\title{
Resonancia magnética en enfermedad de Ménière
}

\author{
Magnetic resonance imaging in Ménière's disease
}

\author{
Maya Kuroiwa R. ${ }^{1,2,3}$, César Fuenzalida R. ${ }^{4}$, Natalia Bahamondes M. ${ }^{4}$, Trung Le $^{5}$
}

\section{Resumen}

La enfermedad de Ménière (EM) es una conocida causa de vértigo crónico asociado a múltiples síntomas audiológicos fluctuantes (hipoacusia, tinnitus, plenitud aural). Su fisiopatología no es completamente comprendida, pero se ha relacionado al hidrops endolinfático (HE) como entidad principal. A la fecha, varios estudios electrofisiológicos han intentado posicionarse como pruebas diagnósticas definitivas, pero no lo han logrado, ya sea por dificultad técnica para realizarlos o por resultados inconsistentes en la literatura internacional. Pero cabe destacar que varios hallazgos son altamente sugerentes de enfermedad de Ménière como la presencia de una baja tasa de respuesta asociado al aumento del umbral de esta misma en los VEMPs (vestibular evoked myogenic potentials), tanto oculares como cervicales, o el aumento del cuociente entre el potencial de sumación/potencial de acción en la electrococleografía. A pesar de estas pruebas, hasta el día de hoy, el diagnóstico se sigue realizando en base a criterios clínicos consensuados internacionalmente, dado lo dificultosa que es la visualización directa del HE y falta de compresión de otros mecanismos fisiopatológicos de esta enfermedad. En las últimas décadas, ha habido avances prometedores en la detección de hidrops endolinfático con el uso de resonancia magnética, permitiendo la visualización de los espacios peri y endolinfáticos usando gadolinio como contraste. A su vez, esto ha permitido mejoría de las secuencias imagenológicas usadas, el desarrollo de varias nuevas técnicas de procesamiento de imágenes, conllevando que varias escalas o clasificaciones de HE hayan sido propuestas, las cuales serán revisadas en detalle en este trabajo.

Palabras clave: Enfermedad de Ménière, resonancia magnética, hidrops endolinfático.

\begin{abstract}
Ménière's disease (MD) is a known cause of chronic vertigo, with multiple fluctuating audiological symptoms (hearing loss, tinnitus, aural fullness). The pathophysiology is not completely understood but has been attributed to endolymphatic hydrops (EH). There are many electrophysiological tests available, but none of them can be considered as the gold standard, either because of difficult technique or inconsistent results in the literature. Nonetheless, some findings are highly suggestive of $M D$, like the presence of a low response and high threshold in ocular and cervical VEMPs (vestibular evoked myogenic potentials), or the increased ratio between the amplitudes of summation potential and action potential in the electrocochleography. Despite the availability of these tests, the diagnosis of $M D$ is still made clinically, following the diagnostic criteria establish by international consensus, due to the difficulty to visualize the $E H$ and also the lack of full comprehension of other pathophysiological mechanisms. In the last decades, there have been promising advances in the detection of endolymphatic hydrops with magnetic resonance imaging, allowing the visualization of the perilymphatic and endolymphatic space using gadolinium as a contrast agent. At the same time, the imaging sequences have improved, new techniques for imaging processing have been developed, entailing that various classifications or graduation systems for EH have been proposed, which will be review in detail, in this paper.
\end{abstract}

Keywords: Menierè's disease, MRI, endolymphatic hydrops.

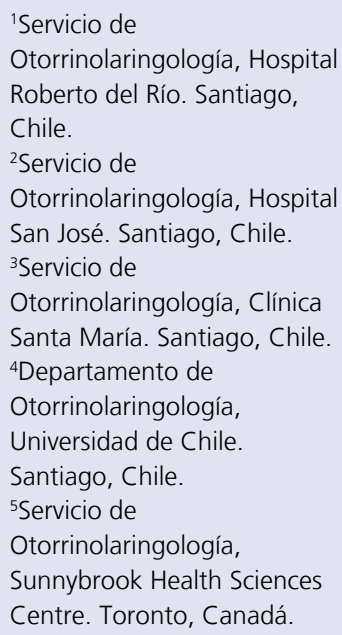

Los autores declaran no tener conflictos de interés.

Recibido el 2 de junio de 2020. Aceptado el 16 de agosto de 2020 .

Correspondencia: Maya Kuroiwa R. Servicio de Otorrinolaringología Clínica Santa María Avenida Santa María 500, Providencia. Santiago, Chile. Email: mkuroiwa@ clinicasantamaría.cl 


\section{Introducción}

La enfermedad de Ménière (EM) es una conocida causa de vértigo crónico, con fluctuaciones de la audición, plenitud aural o auditiva y tinnitus que puede evolucionar a la pérdida progresiva de las funciones audiovestibulares. La fisiopatología de esta enfermedad no está completamente entendida, pero se le ha atribuido un rol primordial al hidrops endolinfático (HE), el cual corresponde a la distensión del espacio endolinfático del oído interno hacia el espacio perilinfático del mismo, ocurriendo principalmente en el conducto coclear y sáculo, pero pudiese afectar al utrículo y los canales semicirculares también ${ }^{1}$.

La causa del hidrops ha sido ampliamente discutida en la literatura y a través de los años, múltiples teorías han tratado de explicar su base fisiopatológica, desde los años 30 en que Hallpike y Cairns describían por primera vez el hidrops, más adelante en los años 60, Schuknecht lo explicaba por una disfunción del saco endolinfático y las crisis vertiginosas por una intoxicación celular por potasio, tras la ruptura de la membrana de Reissner ${ }^{2}$, teoría que ha sido refutada por varios autores, luego Gibson y Arenberg plantearon la "teoría del drenaje" en donde un cambio de flujo radial a longitudinal del líquido endolinfático asociado a un bloqueo del saco endolinfático ${ }^{3}$, provocaría o perpetuaría la EM, por otro lado, para explicar el inicio de este aumento de líquido endolinfático, Hornibrook ha planteado una asociación en la fisiopatología del vértigo postural paroxístico benigno (VPPB) y EM, en esta última otoconias saculares que no son disueltas en saco endolinfático podrían bloquear el saco endolinfático y generar la enfermedad ${ }^{2}$. Si bien, todas estas teorías son interesantes y están en constante desarrollo, no logran explicar la fisiopatología del todo y aún quedan muchas interrogantes que resolver para comprender la EM en su totalidad.

Por otro lado, varios estudios electrofisiológicos han intentado posicionarse como pruebas diagnósticas definitivas de EM, pero no lo han logrado, ya sea por dificultad técnica para realizarlos, baja o variada sensibilidad y por resultados inconsistentes en la literatura internacional. Pero cabe destacar que varios hallazgos son altamente sugerentes de enfer- medad de Ménière como la presencia de una baja tasa de respuesta asociado al aumento del umbral de esta misma en los VEMPs (vestibular evoked myogenic potentials), tanto oculares como cervicales, o el aumento del cuociente entre el potencial de sumación y el potencial de acción del nervio auditivo en la electrococleografía ${ }^{4}$.

A pesar de todo lo descrito, hasta ahora, el diagnóstico de EM es clínico. La Academia Americana de Otorrinolaringología-Cirugía de Cabeza y Cuello (AAO-HNS) ha desarrollado criterios para el diagnóstico desde 1972, estos fueron recientemente modificados en el año 2015, tras una colaboración multinacional de diferentes sociedades donde participó el Comité de Equilibrio de AAO-HNS, la Sociedad de Barany, la Sociedad Japonesa para la Investigación del Equilibrio, entre otras ${ }^{5,6}$. En este consenso se eliminaron las categorías de "cierto" y "posible", sin requerir la confirmación histopatológica de hidrops endolinfático para el diagnóstico de certeza. En la Tabla 1 se resumen los criterios clínicos actuales. Si bien los criterios son claros, cabe destacar que por tener presentación clínica variable y con periodos de remisión, la enfermedad de Ménière puede no presentar todos los síntomas en un comienzo ni tener alteraciones audiométricas y, por lo tanto, el diagnóstico definitivo puede llevar meses e incluso años. Además el HE se ha observado en oídos sanos evaluados por histología post mortem hasta en un $26 \%{ }^{7}$.

Lamentablemente, no hay disponible ningún test o examen específico para realizar el diagnóstico definitivo de EM, esto dado, por la dificultad de objetivar el hidrops endolinfático, complicado aún más por la falta de completa compresión sobre la relación causal entre $\mathrm{HE}$ y EM.

Durante los últimos 10-20 años ha habido prometedores avances en la detección de EM a través de imagenología con resonancia magnética (RM). Uno de los principales desafíos de la imagenología del oído interno, es la habilidad de identificar con certeza el espacio endolinfático. Recordemos que la endolinfa es rica en potasio y baja en sodio, parecido al citosol, en contraste a la perilinfa que es rica en sodio y baja en potasio como el medio extracelular o líquido céfalorraquídeo (LCR). Por lo que solo este último es susceptible de ser realzado 
Tabla 1. Criterios actuales para el diagnóstico de la enfermedad de Ménière

\begin{tabular}{|c|c|}
\hline Definitivo & $\begin{array}{l}\text { - Dos o más episodios de vértigo espontáneo cada uno con una duración de } 20 \text { min a una hora } \\
\text { - Pérdida auditiva neurosensorial en las frecuencias bajas a medias, documentada audiométricamente en } \\
\text { un oído, en al menos una ocasión antes, durante o después de los episodios de vértigo } \\
\text { - Síntomas auditivos fluctuantes (audición, tinnitus, plenitud ótica) en el oído afectado } \\
\text { - Cuadro no se explica mejor por otro diagnóstico vestibular }\end{array}$ \\
\hline Probable & $\begin{array}{l}\text { - Dos o más episodios de vértigo o mareo con una duración de } 20 \text { min a } 24 \text { h } \\
\text { - Síntomas auditivos fluctuantes (audición, tinnitus, plenitud ótica) en el oído afectado } \\
\text { - Cuadro no se explica mejor por otro diagnóstico vestibular }\end{array}$ \\
\hline
\end{tabular}

con contraste. Por otro lado, la membrana de Reissner que divide los espacios o escalas del conducto coclear, es extremadamente delgada y, por tanto, casi imposible de visualizar con la imagenología tradicional ${ }^{8}$.

\section{Secuencias imagenológicas en RM para $\mathrm{HE}$}

En el año 2005, Zou y cols. ${ }^{9}$ fueron capaces de demostrar, en un modelo animal, que el gadolinio (Gd) logra penetrar el oído interno a través de inyección intratimpánica e inyección en ventana redonda. Esto demostró la rápida comunicación entre la ventana redonda y vestíbulo, además entre la escala timpánica y escala vestibular en el conducto coclear. Un par de años después, Nakashima y cols. ${ }^{8}$, usando la secuencia de recuperación de la inversión atenuada de fluido tridimensional (3D-FLAIR) en RM, luego de inyección intratimpánica con gadolinio, logró reportar por primera vez la semicuantificación de $\mathrm{HE}$ en pacientes con EM.

Desde entonces, múltiples estudios han intentado replicar estos resultados, la mayoría usando adquisición retardada de imágenes, luego de inyección intratimpánica (IT) o endovenosa (EV) de $\mathrm{Gd}^{10}$. La vía endovenosa es la más comúnmente usada, ya que es más práctica y menos invasiva, además permite evaluar ambos oídos simultáneamente con una distribución de gadolinio más uniforme y detecta mejor la barrera hemato-perilinfática, en comparación con la inyección intratimpánica $^{11}$. Esta última, si bien es una vía más directa, permitiendo mejor resolución de imágenes, depende también de la permeabilidad de la membrana de la ventana redonda, por lo que es difícil de controlar ${ }^{10}$. Para poder demostrar la baja concentración de Gd en la perilinfa tras la administración endovenosa, se requieren secuencias de RM de alta resolución con una alta relación señal-ruido (SNR. signal to noise ratio), para obtener esto último, el magneto utilizado debe ser de 3 teslas (3T) con una bobina especial para la cabeza y un alto número de canales receptores. Las secuencias más usadas son las de inversión-recuperación atenuada de fluidos, en especial la tridimensional (3D-FLAIR) y también la misma, pero ponderada en T2 (T2W 3D-FLAIR); estas secuencias de inversión-recuperación suprimen las señales de los fluidos, permitiendo la visualización separada de los espacios endolinfáticos de los perilinfáticos, al generar contraste o señal de alta intensidad del espacio perilinfático ${ }^{12}$. El tiempo óptimo para obtener las imágenes, se considera de 24 horas después de la inyección intratimpánica de Gd y entre 4-6 horas luego de la inyección endovenosa de $\mathrm{Gd}^{13}$.

Otra secuencia que ha demostrado utilidad, es la secuencia de inversión-recuperación con reconstrucción real tridimensional (3D-real IR), que permite una mejor diferenciación de la hiperseñal perilinfática de la hiposeñal 
endolinfática y del hueso temporal, lo que permitiría una mejor resolución de la imagen, pero lamentablemente es muy sensible a las concentraciones de Gd en el oído interno, teniendo mejor rendimiento si la inyección de Gd se realiza por vía intratimpánica ${ }^{14}$. Para solucionar este problema, Naganawa y cols. desarrollaron una serie de secuencias, técnicas de posprocesamiento y fusión de imágenes de RM para HE. La sustracción de una imagen positiva de endolinfa desde una imagen positiva de perilinfa fue llamada HYDROPS (HYbriD of Reversed image Of Positive endolymph signal and native image of positive perilymph Signal) ${ }^{15,16} \mathrm{y}$ permite visualizar toda la anatomía del oído interno en una sola serie de imágenes, luego de inyección endovenosa de Gd, sin embargo, la técnica es engorrosa, requiere entrenamiento para ser utilizada, los tiempos de escaneo son muy prolongados y es muy sensible a los movimientos de los pacientes, por lo que no ha tomado la popularidad que se esperaba por parte de los autores.

Por último, están las reconstrucciones volumétricas que se obtienen con cisternografía del oído interno por RM (Gd IT), luego usando secuencias de inversión-recuperación con reconstrucción real tridimensional (3Dreal IR) y algoritmos de aprendizaje automatizado (inteligencia artificial) han logrado generar reconstrucciones con imágenes de muy buena calidad, pero hasta ahora, no se ha logrado crear una escala que permita realizar diagnóstico de EM. Sí podrían tener un rol en monitorización de tratamientos en protocolos de investigación para observar la respuesta y evolución de los sujetos ${ }^{17,18}$.

\section{Clasificación diagnóstica de EM mediante imagenología con RM}

Hasta la fecha, se han propuesto varios sistemas semicuantitativos de graduación del HE para diagnóstico de EM, mediante imágenes de RM. Los revisaremos a continuación.

\section{Clasificación de Nakashima}

Nakashima y colaboradores ${ }^{19}$ proponen un sistema simple de 3 grados para describir HE en la cóclea y vestíbulo (Tabla 2), para esto utilizaron secuencias de imágenes, usando cortes axiales en T2W 3D-FLAIR y 3D real IR, 24 horas posterior a administración de Gadolinio IT. Posterior a esto, se fusionaron ambas imágenes para poder observar más claramente diferencias de perilinfa/endolinfa/ hueso/aire como describe Naganawa y cols. ${ }^{20}$, logrando por primera vez describir hidrops endolinfático en pacientes vivos.

En el vestíbulo, cuando el área del espacio endolinfático es mayor a un tercio del espacio vestibular, se considera como HE. Cuando esta relación excede de 50\%, se considera hidrops significativo. Estas proporciones se proponen debido a la observación de huesos temporales de sujetos sanos, en donde el área del espacio endolinfático fluctúa entre el 26,5\% al 39,4\% (media 33,2\%). En la cóclea, el espacio endolinfático está dividido en las vueltas de la cóclea, en donde la sección medio-modiolar es el mejor espacio para describir los hallazgos. Cuando no hay desplazamiento de la membrana de Reissner, se considera como ausencia de HE, cuando hay desplazamiento

\begin{tabular}{lcl}
\multicolumn{3}{l}{ Tabla 2. Clasificación de Nakashima. Grados de hidrops endolinfático usando RM } \\
\hline Grado de hidrops & Vestíbulo (relación del área)* & Cóclea \\
\hline Sin hidrops & $\leq 33,3 \%$ & Sin desplazamiento de membrana de Reissner \\
\hline Hidrops Leve & $>33,3 \%, \leq 50 \%$ & $\begin{array}{l}\text { Desplazamiento de membrana de Reissner } \\
\text { Área de conducto coclear } \leq \text { área de escala vestibular }\end{array}$ \\
Hidrops significativo & $>50 \%$ & Área de conducto coclear sobrepasa área de escala vestibular
\end{tabular}

* Relación entre el área del espacio endolinfático y el espacio total (suma de espacio perilinfático y espacio endolinfático) en el vestíbulo medido a través de trazado de imágenes. Con autorización de: (adaptado al español): Nakashima T, Naganawa S, Pyykkö I, Gibson WPR, Sone M, Nakata S, Teranishi M. Grading of endolymphatic hydrops using magnetic resonance imaging. Acta Oto-Laryngologica 2009;129(sup560):5-8. 
de la membrana de Reissner, pero el área del conducto coclear es menor o igual al área de la escala vestibular, se considera como hidrops Leve. Cuando el área del conducto coclear sobrepasa el área de la escala vestibular, se considera como hidrops significativo. Esta clasificación coclear considera que cuando el grado de HE difiere entre la espira basal de la cóclea y el resto de las espiras, se clasifica como hidrops significativo.

Algunas limitantes de esta clasificación son:

- El colapso del espacio endolinfático no es posible de reconocer por RM.

- Si hay ruptura de membrana de Reissner, el gadolinio puede entrar en espacio endolinfático, impidiendo realizar la clasificación.

- No diferencia sáculo de utrículo, no son evaluados como entidades separadas.

- Solo 4 huesos temporales de sujetos sanos fueron utilizados para definir los porcentajes mencionados.

- No se ha logrado estandarizar su uso, por el tipo de administración de Gd, por lo que se desconoce la sensibilidad y especificidad de la técnica propuesta.

- La ausencia de hidrops no permite descartar EM 100\%, ya que no siempre significa normalidad.

En el año 2016, Attyé y cols. ${ }^{21}$, usando la clasificación de Nakashima de base, propusieron la medición basada en tamaño y morfología sacular, usando la misma secuencia 3D-FLAIR, llamada radio de inversión sacular utricular (SURI, saccule to utricle ratio). Esto basado en estudios de huesos temporales que muestran que las alteraciones saculares en EM son más frecuentes que aquellas en utrículo ${ }^{22,23}$. SURI fue definida como un radio $\geq 1$ entre el área del sáculo y el área del utrículo en un corte sagital. Así grado 0 es la ausencia de anormalidad sacular, grado 1 SURI y grado 2 sáculo no visible. Este trabajo demostró la presencia de HE solo en pacientes con criterios clínicos de EM y se cree es más confiable que el método semicuantitativo de Nakashima. Sin embargo, esta clasificación solo evalúa el hidrops vestibular, además presenta la dificultad de diferenciar sáculo de utrículo en casos más severos de $\mathrm{HE}^{24} \mathrm{y}$ es muy elaborado el procesamiento de imágenes, ya que se deben reconstruir muchas secuencias para obtener los cortes sagitales necesarios ${ }^{25}$.

\section{Clasificación de Barath}

En el año 2014, Barath y cols. ${ }^{26}$ proponen un sistema de graduación de (HE) de 2 grados, para cóclea y vestíbulo. Para esto, se consideraron 53 pacientes con EM clínicamente confirmada con criterios de la AAO-HNS, todos fueron sometidos a RM 3 Tesla (3T), usando la secuencia 3D-IR con contraste de $\mathrm{Gd}$ endovenoso, a doble de dosis $(0,2 \mathrm{mmol} /$ $\mathrm{kg}$ ) inyectado 4 horas previo a la toma de imágenes. En las imágenes obtenidas a través de esta secuencia (3D-IR), fue posible identificar: el septum interescalar, escala timpánica, conducto coclear/lámina espiral ósea y escala vestibular. En un vestíbulo normal, las áreas agregadas del sáculo y utrículo son menos que la mitad del área del vestíbulo en el nivel mediomodiolar (Figura 1A).

\section{Graduación coclear}

- HE coclear grado I: Dilatación leve de la zona del conducto coclear no realzada, preservando partes de la zona realzada de escala vestibular (Figura 1B).

- HE coclear grado II: Escala vestibular obstruida uniformemente por una distensión máxima del conducto coclear (Figura 1C).

\section{Graduación vestibular}

- HE vestibular Grado I: Distensión > 50\% del espacio endolinfático del sáculo utrículo o ambos, con el espacio perilinfático aún visible a través de la periferia del vestíbulo óseo (Figura 1B, Figura 2).

- HE vestibular grado II: Vestíbulo óseo abarcado completamente por espacio endolinfático dilatado (Figura 1C).

Este sistema de graduación, en el trabajo original $^{26}$, mostró una correlación de $90 \%$ entre la presencia de $\mathrm{EH}$ en los oídos estudiados de pacientes con síntomas clínicos de EM. A su vez, $78 \%$ de los oídos sanos no mostraron HE. Por lo que el coeficiente de correlación de Spearman entre la presencia de EH y la clasificación clínica fue de 0,67 . Esta clasificación, si bien permite obtener resultados más específicos y confiables que la graduación de Nakashima, tampoco entrega niveles de sensibilidad ni especificidad. 


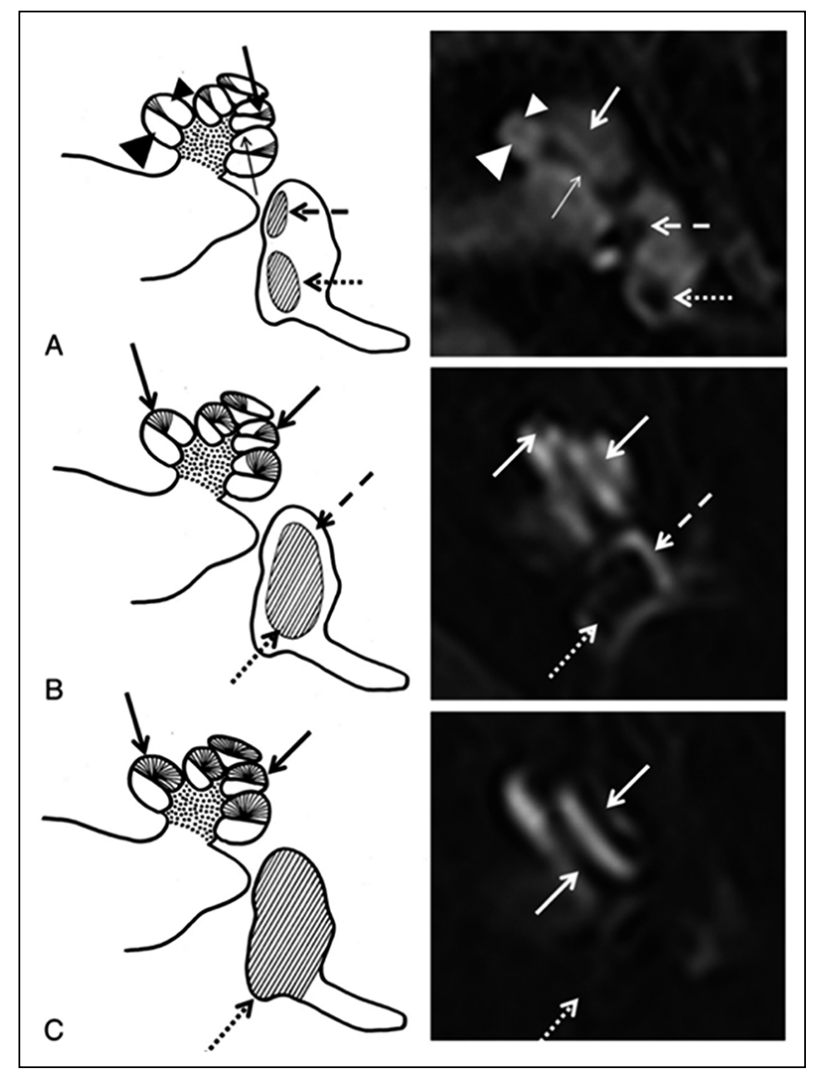

Figura 1. (A) Laberinto normal: Septum interescalar (flecha delgada), escala timpánica (cabeza de flecha grande), conducto coclear/lámina espiral ósea (flecha gruesa), escala vestibular (cabeza de flecha pequeña), sáculo (flecha discontinua) y utrículo (flecha punteada). (B) Hidrops coclear grado I con dilatación irregular y obstrucción parcial de escala vestibular (flechas). En hidrops vestibular grado I, dilatación del espacio endolinfático (flechas punteadas) abarca $>50 \%$ del vestíbulo. Un espacio perilinfático circular (flecha discontinua) permanece visible. (C) Hidrops coclear grado II con obliteración total de la escala vestibular (flechas). En hidrops vestibular grado II, espacio endolinfático dilatado ocupa completamente el vestíbulo. Con autorización de: Baráth y cols. Detection and Grading of Endolymphatic Hydrops in Menière Disease Using MR Imaging. Am J Neuroradiol 2014;35(7):1387-92, doi: 10.3174/ ajnr. A3856.

\section{Clasificación de Bernaerts}

El sistema de graduación de Barath ${ }^{26}$ es revisado por Bernaerts en el año 2019, agregando la definición clínica actual de EM propuesta en el año $2015^{5}$. En esta nueva graduación se utilizó el mismo protocolo de procesamiento y secuencias de imágenes en la RM que Barath, siendo además, la serie más grande hasta la fecha, incluyendo 148 pacientes con EM. Utilizó el oído contralateral del paciente operado como control. Con relación a los grados de hidrops coclear (Figura 3), Bernaerts mantiene la misma clasificación original, pero en el vestíbulo se agrega un grado previo, debido a que existían pacientes con pequeñas anormalidades clasificados como normales en la clasificación de Barath, obteniendo lo siguiente: HE vestibular grado I definido como sáculo igual o mayor a utrículo, sin presentar confluencia con utrículo. Grado II igual a grado I de Barath, y grado III igual que grado II de Barath (Figura 4).

Se revisó también semicuantitativamente, el grado de realce de perilinfa coclear y vestibular por separado, clasificándose en: menor, igual o mayor a oído contralateral sustentado en la teoría de que EM produce aumento de permeabilidad de barrera hemato-perilinfática ${ }^{27}$. Se encontró que el realce perilinfático coclear (RPC) es el parámetro de mayor valor predictivo para definir EM definitiva (Figura 5), y en conjunto con la presencia de HE vestibular de 4 grados se obtienen niveles de sensibilidad y especificidad de $84,6 \%$ y $92,3 \%$ respectivamente.

Además, el agregar el cuarto grado vestibular (tamaño sacular), aumenta la sensibilidad y sin bajar la especificidad; esto

Figura 2. Dilatación sacular predominante: secuencia 3D-IR (oídos derecho e izquierdo del mismo paciente). El lado izquierdo muestra un sáculo dilatado (flecha) y un utrículo levemente dilatado (flecha discontinua) con HE coclear grado I (flecha punteada). Se nota el incremento en el realce de la perilinfa en el oído enfermo vs oído normal (derecha). Con autorización de: Baráth y cols. Detection and Grading of Endolymphatic Hydrops in Menière Disease Using MR Imaging. Am J Neuroradiol. 2014;35(7):1387-92, doi: 10.3174/ajnr. A3856.

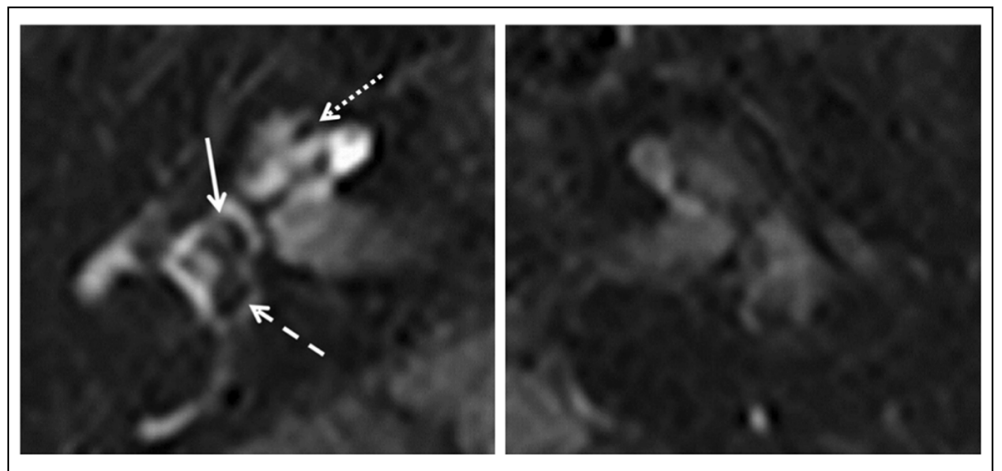



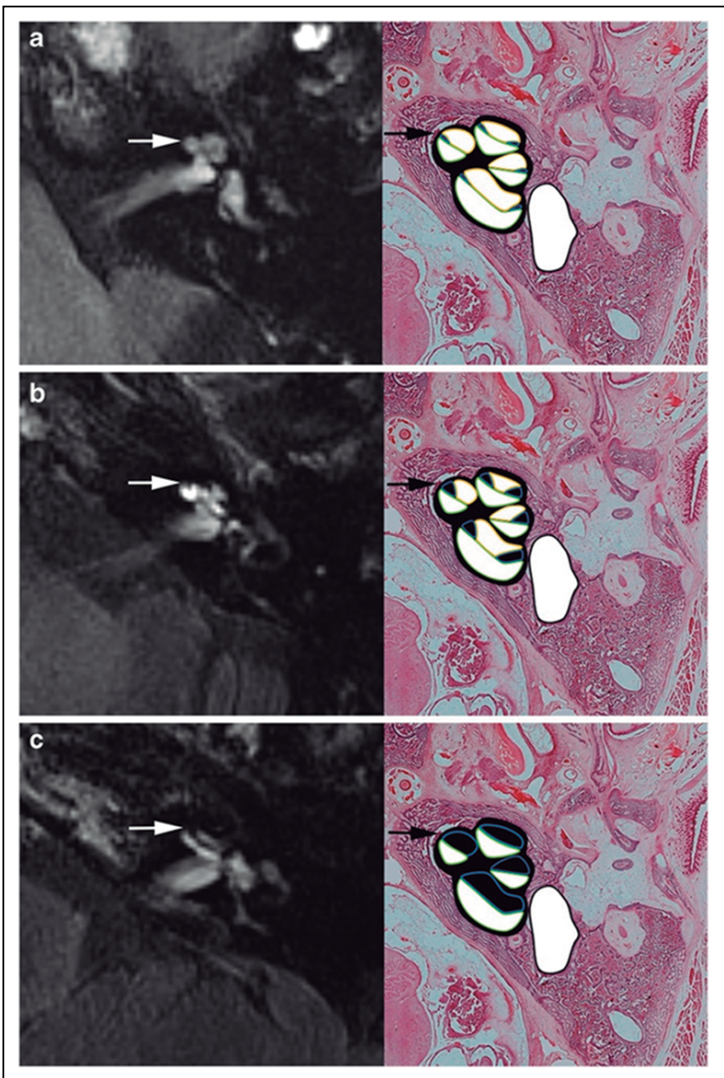

Figura 3. Imágenes axiales 3D FLAIR, obtenidas luego de Gd EV Cortes del área mediomodiolar de la cóclea y sus correlativos en criosecciones teñidas con hematoxilina-eosina (magnificación x7). a: Cóclea normal: se reconoce septum interescalar (flecha), escala timpánica y escala vestibular. La escala media es normalmente mínimamente visible. b: Hidrops coclear grado I: La escala media es visible indirectamente como una zona negra recortada de la escala vestibular (flecha). c: Hidrops coclear grado II: La escala vestibular (flecha) está completamente obliterada por la distensión del conducto coclear. Con autorización de: Bernaerts y cols. The Value of Four Stage Vestibular Hydrops Grading and Asymmetric Perilymphatic Enhancement in the Diagnosis of Menière's Disease on MRI. Neuroradiology 2019; 61(4):421-29, doi: 10.1007/ s00234-019-02155-7.

concuerda con la descripción de SURI, por Attyé y cols. ${ }^{21}$, como un marcador importante en EM. El resto de las variables como HE coclear, o realce perilinfático vestibular no aportan a la discriminación de las imágenes. Debido a esto, en conjunto con proponer un sistema de clasificación de HE coclear y vestibular modificado, se propone un algoritmo de estudio para EM unilateral (Figura 6). Algunas limitaciones de este estudio fue usar el oído contralateral sano de los mismos sujetos,
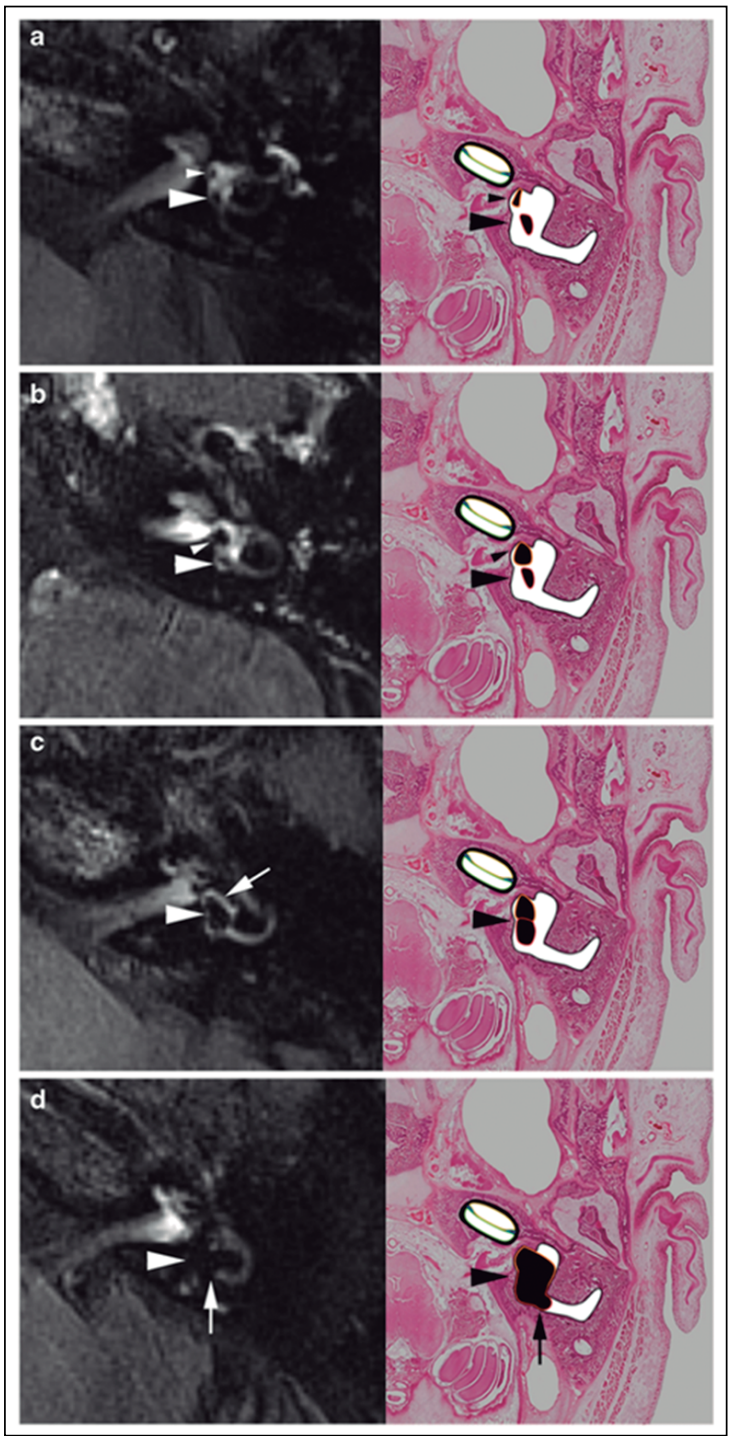

Figura 4. Imágenes ponderadas en 3D-FLAIR cortes axiales de la parte inferior del vestíbulo, en comparación con la misma sección en un preparado de hematoxilina-eosina. a: Vestíbulo normal: El sáculo (cabeza de flecha pequeña) y utrículo (cabeza de flecha grande) son visualizados por separado, y ocupan menos del 50\% del vestíbulo. b: Hidrops vestibular grado I: El sáculo (cabeza de flecha pequeña), normalmente el de menor tamaño de los sacos, se vuelve igual o más grande que el utrículo (cabeza de flecha grande), pero aún no confluye con el utrículo. c: Hidrops vestibular grado II: Existe confluencia de sáculo/utrículo (cabeza de flecha), pero aún se observa un realce de perilinfa alrededor. d: Hidrops vestibular grado III: El realce perilinfático no es observable (cabeza de flecha). Hay una obliteración total del vestíbulo óseo. Se observa también en este caso, el inicio de la protrusión utricular en el brazo no ampular del CSC lateral (flecha). Con autorización de: Bernaerts y cols. The Value of Four Stage Vestibular Hydrops Grading and Asymmetric Perilymphatic Enhancement in the Diagnosis of Menière's Disease on MRI. Neuroradiology 2019; 61(4):421-29, doi: 10. 1007/s00234-019-02155-7. 
pero lamentablemente todos los estudios utilizan el mismo método, ya que es poco ético someter a pacientes sanos a RM con contraste, solo para ver oídos sanos.

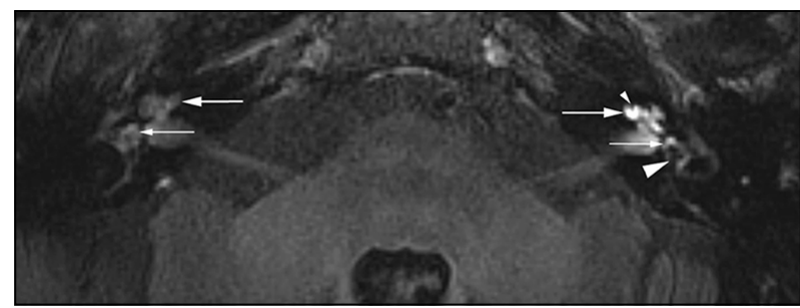

Figura 5. RM 3D-FLAIR con gadolinio. Cortes axiales de una mujer de 77 años con EM unilateral definitiva e HE coclear grado I (cabeza de flecha pequeña), HE vestibular grado II de acuerdo a la clasificación de 4 etapas (cabeza de flecha grande). Se observa el aumento del realce perilinfático vestibular (flecha pequeña) y coclear (flecha grande) en el oído enfermo, en comparación con el oído sano. Este es el signo que corrobora la permeabilidad de la barrera sangre-perilinfa. Con autorización de: Bernaerts y cols. "The Value of Four Stage Vestibular Hydrops Grading and Asymmetric Perilymphatic Enhancement in the Diagnosis of Menière's Disease on MRI" Neuroradiology 2019; 61(4):421-29, doi:10. 1007/s00234-019-02155-7.

\section{Conclusión}

La imagenología, sin lugar a duda, ha avanzado muy rápido durante las últimas décadas. Los procesamientos de imágenes cada vez son más sofisticados y nos permiten obtener buenas aproximaciones de lo que realmente ocurre en el oído interno. Pero como fue discutido con anterioridad, el tamaño, ubicación y características de fluidos en la cóclea y sistema vestibular, hacen que la obtención de imágenes sea un desafío. Muchos grupos de investigación están trabajando en encontrar una clasificación que nos permita un diagnóstico más certero de la EM.

Aunque ya existen varias que nos otorgan una gran ayuda, como revisamos en este trabajo; la discrepancia clínico-radiológica aún refleja que hay un incompleto conocimiento de la fisiopatología de la EM, sabiendo, además, que entre $10 \%$ a $33 \%$ de los pacientes con EM no tienen cambios demostrables (HE) a la RM ${ }^{27,28}$. Es probable que en la medida que la ciencia

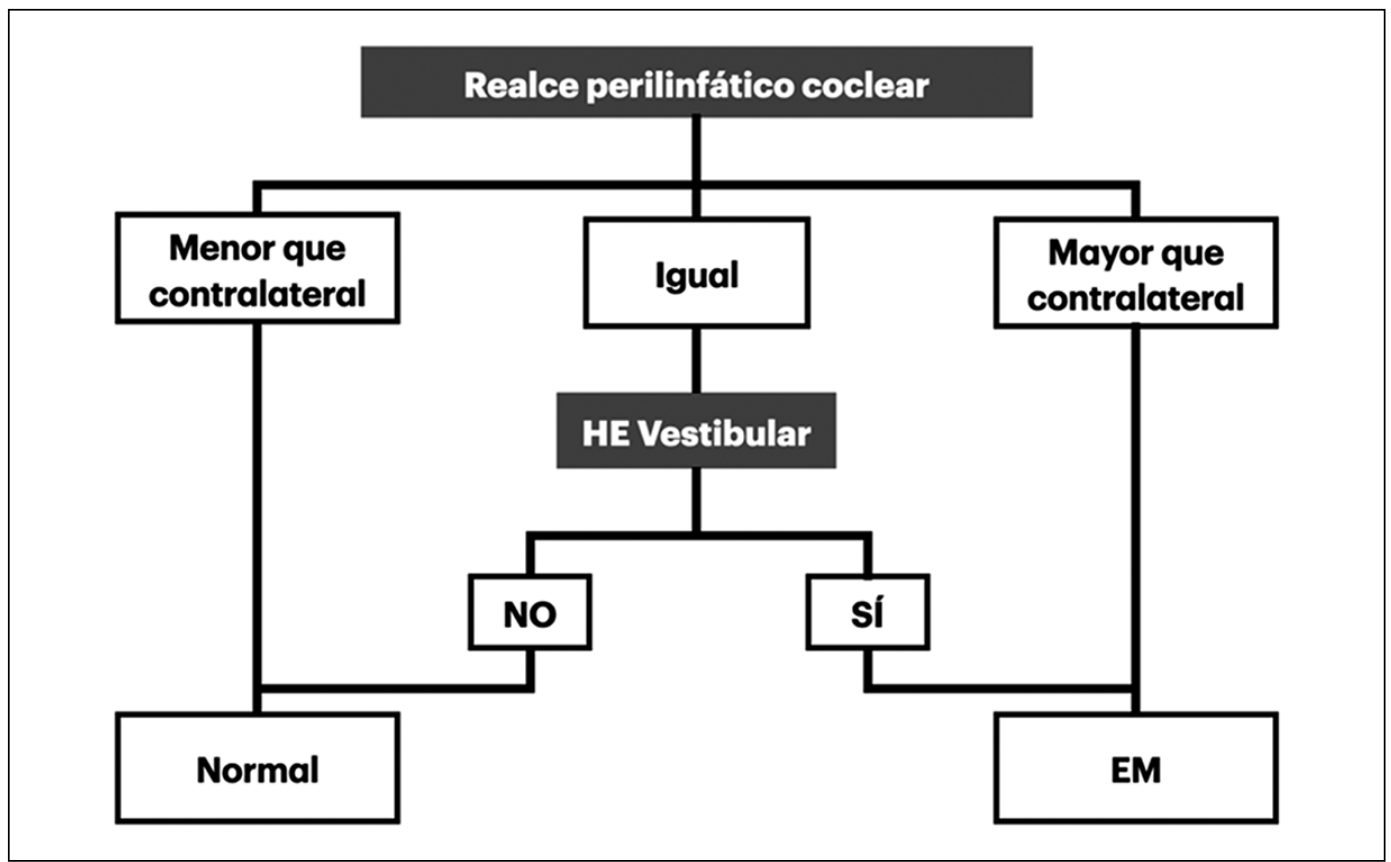

Figura 6. Algoritmo propuesto para EM unilateral. Si el realce perilinfático coclear (RPC) es menor que el contralateral, el oído puede ser considerado normal, si es igual, se puede considerar normal si no existe HE vestibular. Por otro lado, si existe HE vestibular, es altamente sospechoso de tener EM. Si el RPC es mayor al contralateral, el oído puede ser clasificado como EM positiva, independiente de la ausencia o presencia de HE vestibular. Adaptado con permiso de: Bernaerts y cols. "The Value of Four Stage Vestibular Hydrops Grading and Asymmetric Perilymphatic Enhancement in the Diagnosis of Menière's Disease on MRI" Neuroradiology 2019;61(4):421-29, doi:10. 1007/s00234-019-02155-7. 
nos permita comprender con mayor claridad la fisiopatología, con mejor identificación de agentes causales o gatillantes, la dirección futura de las investigaciones se enfocará a la imagenología asociada a biomarcadores de actividad de la EM, expandiendo la visión de solo buscar HE.

\section{Bibliografía}

1. Gürkov R, Pyykö I, Zou J, Kentala E. What is Ménière's disease? A contemporary re-evaluation of endolymphatic hydrops. Journal of Neurology. 2015;263(1):71-81. doi: 10.1007/s00415-015-7930-1.

2. Hornibrook J. Saccular otoconia as a cause of Ménière's disease: hypothesis based on two theories. J Laryngol Otol. 2018;132(9):771-774. doi: 10.1017/ S0022215118001366.

3. FRACS WPRGMF. Hypothetical Mechanism for Vertigo in Ménière's Disease. Otolaryngologic Clinics of NA. 2010;43(5):1019-1027. doi: 10.1016/j. otc.2010.05.013.

4. Lamounier P. Electrophysiology in Ménière's Disease. In: Up to Date on Meniere's Disease. InTech; 2017:116. doi: 10.5772/intechopen.69668.

5. Goebel JA. 2015 Equilibrium Committee Amendment to the 1995 AAO-HNS Guidelines for the Definition of Ménière's Disease. Otolaryngol Head Neck Surg. 2016;154(3):403-404. doi: $10.1177 / 0194599816628524$.

6. López-Escamez JA, Carey J, Chung W-H, et al. Diagnostic criteria for Ménière's disease. VES. 2015;25(1):1-7. doi: 10.3233/VES-150549.

7. Yamazaki M, Naganawa S, Tagaya M, et al. Comparison of Contrast Effect on the Cochlear Perilymph after Intratympanic and Intravenous Gadolinium Injection. AJNR Am J Neuroradiol. 2012;33(4):773-778. doi: 10.3174/ajnr.A2821.

8. Nakashima T, Naganawa $S$, Sugiura $M$, et al. Visualization of Endolymphatic Hydrops in Patients With Ménière's Disease. The Laryngoscope. 2007;117(3):415-420. doi: 10.1097/ MLG.0b013e31802c300c.

9. Zou J, Pyykkö I, Bjelke B, Dastidar P, Toppila E. Communication between the Perilymphatic Scalae and Spiral Ligament Visualized by in vivo MRI. Audiol Neurotol. 2005;10(3):145-152. doi: $10.1159 / 000084024$.

10. Lingam RK, Connor SEJ, Casselman JW, Beale T. MRI in otology: applications in cholesteatoma and Ménière's disease. Clinical Radiology. September 2017:1-10. doi: 10.1016/j.crad.2017.09.002.

11. Loureiro RM, Sumi DV, Tames HL de VC, et al. Endolymphatic hydrops evaluation on MRI: Practical considerations. American Journal of
Otolaryngology. 2020;41(2):102361-15. doi: 10.1016/j. amjoto.2019.102361.

12. Widmann G, Henninger B, Kremser C, Jaschke W. MRI Sequences in Head \& Neck Radiology-State of the Art. Fortschr Röntgenstr. 2017;189(05):413-422. doi: 10.1055/s-0043-103280.

13. Naganawa S, Nakashima T. Visualization of endolymphatic hydrops with MR imaging in patients with Ménière's disease and related pathologies: current status of its methods and clinical significance. Jpn J Radiol. 2014;32(4):191-204. doi: 10.1007/ s11604-014-0290-4.

14. Conte G, Caschera L, Calloni S, et al. MR Imaging in Ménière Disease: Is the Contact between the Vestibular Endolymphatic Space and the Oval Window a Reliable Biomarker? AJNR Am J Neuroradiol. 2018;39(11):2114-2119. doi: 10.3174/ ajnr.A5841.

15. Naganawa S, Yamazaki M, Kawai H, Bokura K, Sone M, Nakashima T. Imaging of Ménière's Disease after Intravenous Administration of Single-dose Gadodiamide: Utility of Multiplication of MR Cisternography and HYDROPS Image. MRMS. 2013;12(1):63-68. doi: 10.2463/mrms.20120027.

16. Naganawa S, Yamazaki M, Kawai H, et al. MR Imaging of Ménière's Disease after Combined Intratympanic and Intravenous Injection of Gadolinium using HYDROPS2. MRMS. 2014;13(2):133-137. doi: 10.2463/mrms.2013-0061.

17. Gürkov R, Berman A, Dietrich O, et al. MR volumetric assessment of endolymphatic hydrops. Eur Radiol. 2014;25(2):585-595. doi: 10.1007/s00330014-3414-4.

18. Ito $\mathrm{T}$, Inui $\mathrm{H}$, Miyasaka $\mathrm{T}$, et al. Endolymphatic volume in patients with meniere's disease and healthy controls: Three dimensional analysis with magnetic resonance imaging. Laryngoscope Investigative Otolaryngology. 2019;4(6):653-658. doi: 10.1002/ lio2.313.

19. Nakashima T, Naganawa S, Pyykkö I, et al. Grading of endolymphatic hydrops using magnetic resonance imaging. Acta Oto-Laryngologica. 2009;129(sup560):58. doi: 10.1080/00016480902729827.

20. Naganawa S, Sugiura M, Kawamura M, Fukatsu H, Sone M, Nakashima T. Imaging of Endolymphatic and Perilymphatic Fluid at 3T After Intratympanic Administration of Gadolinium-DiethyleneTriamine Pentaacetic Acid. AJNR Am J Neuroradiol. 2008;29(4):724-726. doi: 10.3174/ajnr.A0894.

21. Attyé A, Eliezer M, Boudiaf N, et al. MRI of endolymphatic hydrops in patients with Meniere's disease: a case-controlled study with a simplified classification based on saccular morphology. Eur Radiol. December 2016:1-9. doi: 10.1007/s00330-0164701-z.

22. Rauch SD, Merchant SN, Thedinger BA. Ménière’s 
Syndrome and Endolymphatic Hydrops. Ann Otol Rhinol Laryngol. 2016;98(11):873-883. doi: 10.1177/000348948909801108.

23. Morita N, Kariya S, Farajzadeh Deroee A, et al. Membranous labyrinth volumes in normal ears and Ménière disease: A three-dimensional reconstruction study. The Laryngoscope. 2009;119(11):2216-2220. doi: 10.1002/lary.20723.

24. López-Escamez JA, Attyé A. Systematic review of magnetic resonance imaging for diagnosis of Meniere disease. VES. 2018;29(2-3):121-129. doi: 10.3233/ VES-180646.

25. Bernaerts A, de Foer B. Imaging of Ménière Disease. Neuroimaging Clin N Am. 2019;29(1):19-28. doi: 10.1016/j.nic.2018.09.002.

26. Baráth K, Schuknecht B, Naldi AM, Schrepfer
T, Bockisch CJ, Hegemann SCA. Detection and Grading of Endolymphatic Hydrops in Ménière Disease Using MR Imaging. AJNR Am J Neuroradiol. 2014;35(7):1387-1392. doi: 10.3174/ajnr.A3856.

27. Pakdaman MN, Ishiyama G, Ishiyama A, et al. Blood-Labyrinth Barrier Permeability in Ménière Disease and Idiopathic Sudden Sensorineural Hearing Loss: Findings on Delayed Postcontrast 3D-FLAIR MRI. AJNR Am J Neuroradiol. 2016;37(10):19031908. doi: 10.3174/ajnr.A4822.

28. Pyykkö I, Nakashima T, YOSHIDA T, Zou J, Naganawa S. Ménière's disease: a reappraisal supported by a variable latency of symptoms and the MRI visualisation of endolymphatic hydrops. BMJ Open. 2013;3(2):e001555-11. doi: 10.1136/ bmjopen-2012-001555. 